\title{
Conscience verte et éducation au Burkina Faso «Nous abandonnons cela en soulevant les seaux »
}

\author{
Constance Lavoie (Université du Québec à Chicoutimi) \\ Amélie Baillargeon (Université de Montréal)
}

\begin{abstract}
Résumé
Cet article examine l'intégration d'une « conscience verte » chez les élèves d'écoles primaires en fonction de deux types d'éducation : bilingue ou unilingue. Dix des anciens interviewés sont diplômés de l'école unilingue, dont le curriculum est né pendant la colonisation, tandis que les dix autres sont allés à l'école bilingue où savoirs, savoir-faire et langues autochtones font partie des activités scolaires.

L'article présente les principales préoccupations environnementales de vingt villageois burkinabè sous les angles de la santé, du genre, de la politique et de l'éducation ainsi que les pistes de réflexion qu'ils proposent. À partir des témoignages des interviewés, les auteures survolent les questions de l'urbanisme, de l'eau, de la déforestation, de l'alimentation, des maladies, des conditions de vie des femmes, du transfert des connaissances, de la préservation des langues autochtones, de la hiérarchie, de la protection de l'environnement ainsi que du rôle de l'éducation à ces égards.
\end{abstract}

\begin{abstract}
This article examines the integration of a « conscience verte " (ecological sense) among primary level students who received two different types of schooling :-- bilingual and uniligual. Ten of those interviewed were from a unilingual school whose curriculum dates to the time of colonization, whereas ten other students went to a bilingual school where knowledge, savoir-faire, and indigenous languages are part of the scholarly activities.

The article presents the principal environmental preoccupations of the 20 students from Burkina Faso regarding issues of health, gender, policy and education as well as their opinions on how to address them. According to the reports of those interviewed, the authors consider questions of urbanization, of water, deforestation, nutrition, illnesses, the living conditions of women, the transfer of knowledge, preservation of indigenous languages, hierarchies and protection of the environment as well as the role of education to address these issues.
\end{abstract}

En 1987, le rapport Brundtland a soulevé un débat de fond sur l'environnement et le développement en indiquant l'urgence de considérer la planète comme un seul écosystème (Babin \& Vaillancourt; Benneh, Morgan, \& Uitto, 1996). La menace qui plane sur l'environnement n'est donc pas un phénomène nouveau. Pendant la colonisation, la pression environnementale de l'homme sur la nature a atteint des proportions importantes dues à une exploitation intensive de la faune et de la flore (Chouaibou Mfenjou, 2002). C'est durant la colonisation qu'ont également émergé les premières normes internationales de protection des ressources naturelles, illustrant ainsi les premiers balbutiements d'une prise de conscience à l'égard de la finitude des ressources (Chouaïbou Mfenjou, 2002). 
Des mesures ont ainsi été prises pour cibler les espèces en voie de disparition et restreindre la chasse. Cependant, elles étaient nettement insuffisantes, car aujourd'hui, les effets du pillage se font plus que jamais sentir entre autres en Afrique. L'urgence d'agir a même atteint les établissements d'enseignement : on voit notamment apparaitre de plus en plus dans les curricula scolaires cette « conscience verte " (Jenkins \& Jenkins, 2005). Par conscience verte, on entend le souci accordé à la protection des écosystèmes et à la préservation de la biodiversité (O’Brien, 1997a). Les enfants apprennent donc l'importance de protéger les écosystèmes et leur biodiversité (Cajete, 1999; Rose, 2005). Pour sa part, Orr (1992) utilise plutôt le synonyme " littératie écologique ${ }^{1}$, qu'il définit comme l'habileté à se questionner sur l'incidence à long terme des actions posées sur son environnement et sur celui des autres. La littératie écologique dépassant les murs de la classe, elle se traduit par des compétences et une sensibilité transférables dans tous les domaines de la vie de l'enfant et de l'adulte.

La littérature est sans équivoque : plus une personne est instruite, plus elle accorde de l'importance à l'environnement qui l'entoure (Hanley, 2005; O'Brien, 1997b; Yoshizumi \& Takaaki, 2005). Toutefois, on peut se poser la question à savoir si tous les types d'éducation ont la même incidence sur le développement de la conscience verte. L'intention de départ de cet article était d'illustrer la relation qu'entretiennent les villageois à l'égard de l'environnement et de déterminer si le type d'éducation reçue, unilingue ${ }^{2}$ ou bilingue ${ }^{3}$, exerce une influence sur leur niveau d'engagement et d'appropriation du milieu.

Bien que les chercheures aient passé plus de deux ans dans ce pays, elles ne sont pas burkinabè ${ }^{4}$. Le lecteur doit considérer leur subjectivité à la lecture du présent article. Elles ont choisi les deux villages où ont débuté les écoles bilingues afin de trouver des adultes aptes à développer une réflexion sur l'incidence de leur éducation primaire. Ces villages mossi comptent respectivement 1494 et 1655 habitants (Kibora, Napon, Batiana, \& Belemgoabga, 1999), et l'agriculture est leur principale activité économique. Vingt anciens élèves des écoles bilingue et unilingue, aujourd'hui âgés entre 20 et 40 ans, témoignent du lien qu'ils tissent entre l'éducation reçue et l'environnement. Pour les besoins de l'article, les anciens élèves de l'école bilingue portent des noms burkinabè tandis que ceux de l'école unilingue se sont vus attribués des noms «francophones ». Entre parenthèses se trouve l'âge du participant lors de l'entretien. Par exemple, Pousga (27) est une femme de 27

\footnotetext{
${ }^{1}$ Ecological literacy (Traduction libre, Constance Lavoie)

${ }^{2}$ L'éducation unilingue réfère à l'instruction donnée uniquement en français, langue étrangère enseignée comme langue première.

${ }^{3}$ L'éducation bilingue réfère à l'enseignement transitionnel de la langue du milieu de l'enfant vers le français. Le français est enseigné comme langue seconde. La seconde spécificité de l'éducation bilingue est qu'elle inclut des activités culturelles, économiques et environnementales propres au milieu de vie de l'enfant. Ces activités sont offertes par les parents et les aînés de la collectivité.

${ }^{4}$ Les noms et les adjectifs « burkinabè » et « mossi » restent toujours invariables.
} 
ans ayant étudié dans une école bilingue tandis que Fabrice (21), âgé de 21 ans, est un ancien élève de l'école unilingue.

L'hypothèse propose que les anciens de l'école bilingue, dont le curriculum inclut des activités économiques et culturelles endogènes, sont davantage en mesure de relever les problèmes environnementaux et les solutions qui s'y rattachent. Toutefois, les données recueillies tirées des vingt entretiens semi-dirigés ne permettent pas de tirer de conclusions à l'égard de cette hypothèse. Aux questions sur les problèmes environnementaux dans le village des participants et les solutions envisagées, nous avons obtenu une variété de réponses reflétant leurs préoccupations. Nous avons alors réalisé que le terme « environnement » est polysémique et se rattache à différents domaines de vie, tels que la santé, le genre, la politique et l'éducation. Les entretiens avec les participants permettent d'amorcer une discussion et de déterminer des pistes de réflexion sur la corrélation entre environnement, langue et éducation. C'est pour cette raison que cet article suit cette structure thématique.

\section{ENVIRONNEMENT ET SANTÉ}

Étroitement liés, l'environnement et la santé sont deux réalités souvent défaillantes dans les pays en développement. Ressources mal gérées faute de moyens appropriés, quantités d'aliments insuffisantes découlant de conditions précaires de production, maladies se multipliant à cause d'un manque de salubrité, ce ne sont là que quelques exemples des conséquences des problèmes de santé associés à l'environnement relevés par les participants.

En déclarant «Étant donné que la localité a été lotie, il y a des gens qui viennent s'installer ajouter donc le problème d'eau se pose », Mathieu (38) parle du phénomène de la pression démographique sur l'accès aux ressources. Cette problématique est répandue dans les pays en développement. Lorsqu'une zone est lotie, elle est souvent déjà surpeuplée, puisque les moins nantis y élisent domicile bien avant le lotissement, dans l'attente et l'espoir qu'un jour, le gouvernement choisira d'y installer les infrastructures nécessaires pour vivre dignement. Toutefois, il arrive souvent que le gouvernement les exproprie lorsqu'il lotit une zone. Ce fut le cas pour les résidents de Ouaga 2000 où siègent désormais les bureaux des ministres et les maisons de l'élite. Selon les données de l'Organisation Mondiale de la Santé de 2006, 66 \% des habitants en zone rurale du Burkina Faso ont accès à des « sources d'eau améliorées », contre $97 \%$ pour les citadins (OMS, 2006). Onze des vingt participants identifient l'accès à l'eau comme le principal problème de leur environnement. Le manque de puits et de barrages ainsi que la maintenance de ceux-ci sont aussi très problématiques :

Dans notre quartier ici, c'est que les puits qui sont ici, les gens souffrent du problème d'eau. Même à l'école nous voyons que les élèves souvent même, ont des problèmes avec l'eau. Tônd saka pùg kaane, yaa tà bulsâ sên be kaane wâ, nebâ 
namesda wùsg ne koomâ yelle. Ba ekollê wâ menga, tônd getam tà karenbiisâ sasa ninga, b tara zu-loees ne kooma yelle. » (Yves (29))

Au Burkina Faso, de nombreuses années peuvent s'écouler avant qu'une zone périurbaine de la capitale soit lotie. Une fois la zone lotie par contre, les gens deviennent propriétaires de leur parcelle s'ils ont les moyens de l'acheter et jouissent éventuellement de services comme l'accès à l'eau ou à l'électricité. Fabrice (21) explique que le lotissement incite les gens à vendre la terre ancestrale pour faire de l'argent :

Concernant l'environnement ce que j'ai à dire c'est le fait les gens vendent la terre. Certains vendent la terre de leurs pères. Mam sẽn tar gomd sẽn tõe $n$ togs beene yaa wala nebã sẽn kood tẽn-gãoongã. Nina mi $n$ wa yãka a ba pv-wegga $n$ koose.

La gestion des déchets est une autre composante de la santé publique mentionnée par Yves (29) : «(...), le fait qu'on jette au hasard les papiers, tout cela est quelque chose qui tue notre environnement ». Amélie (23) rajoute qu'il manque d'infrastructures sanitaires pour gérer les excréments humains et que, par conséquent, les gens vont dans la brousse pour faire leurs besoins. Ainsi, il devient difficile d'assurer la préservation de l'environnement. Les données compilées par l'OMS en 2006 pour le Burkina Faso corroborent le fait que de telles infrastructures soient largement absentes : seuls $6 \%$ des habitants en zone rurale ont accès à des systèmes d'assainissement améliorés comparativement à $41 \%$ en zone urbaine (OMS, 2006). Cette situation a donc une incidence directe sur l'eau, la salubrité et conséquemment la santé, puisque de nombreuses maladies, tel le paludisme, sont liées à l'eau.

La qualité déficiente des routes constitue un autre problème environnemental lié à la santé dont Marie (24) et Jean (27) font mention. Toutefois, ils ne le lient pas à la santé, mais nous tenions à souligner les conséquences de ce problème sur la santé des gens. L'omniprésence de routes de poussière entraîne des maladies et des risques d'épidémie, notamment lorsque l'harmattan souffle, vent de la saison sèche qui soulève la poussière porteuse de la méningite. En 2002, le Burkina Faso, pays de la « ceinture de la méningite », a été frappé par une épidémie du sérogroupe W135 du méningocoque, bactérie responsable de la cette maladie : environ 13000 Burkinabè en sont morts. Par l'exemple du Malawi, Moyo (2003) présente l'importance de la publication et de la diffusion de l'information concernant la santé dans les autres langues nationales pour rejoindre l'auditoire le plus vaste possible :

(...) dans les programmes portant sur la prévention de maladies, comme la malaria ou la tuberculose, les langues locales seraient choisies pour atteindre les communautés ciblées. (...) Elles doivent être reconnues pour le rôle crucial 
qu'elles jouent sur divers plans dans le développement national en tant que langues d'enseignement et moyens de communication relativement aux activités liées à l'agriculture, à la santé et à l'économie domestique, activités influant sur la vie des villageois. ${ }^{5}$ (p. 35)

De plus, comme le suggère Omoniyi (2003), l'intégration des savoirs autochtones en matière de traitements médicinaux au curriculum scolaire permettrait aux villageois d'éviter de les oublier avec le temps et d'accéder plus facilement à des traitements :

(...) de considérables contributions pourraient être apportées au développement si les herboristes locaux disposaient des outils de littératie dont ils ont besoin pour documenter et diffuser l'information sur les fonctions curatives transmises oralement des générations précédentes $(\ldots)^{6}$. (p. 146)

Indirectement liée à la santé, la déforestation pose problème à l'égard de l'alimentation et de l'agriculture. Les femmes doivent couper le bois pour cuisiner, puisque seuls $7 \%$ des habitations du Burkina Faso disposent d'électricité (PNUD, 2007). En outre, selon le Rapport sur le développement humain 2007-2008, entre 1990 et 2005, la surface boisée a diminué de $3600 \mathrm{~km}$ carrés, ce que signale Amélie (23) en disant : « Mais de plus en plus la brousse s'en va». Yves (29) ajoute que la déforestation découle de l'activité de l'homme : «... de la manière dont on coupe les arbres, ça aussi ça dégrade notre environnement. le paasd me, yaa tà tààsâ $n$ sên kèè tààsâ modèl ninga wâ, yê me le sâamd tônd têngâongâ. ». En réponse à une telle situation, un groupe voulant préserver les forêts tout en ne privant pas les femmes de cette ressource multifonctionnelle a vu le jour au Kenya : le Green Belt Movement, lauréat du prix Nobel de la Paix en 2004. La fondatrice, Wangari Maathai (2001), note le rôle fondamental du bois dans le régime alimentaire des familles rurales de l'Afrique :

(...), quand ils sont rares, ces combustibles doivent être utilisés avec parcimonie. Ainsi, (...), les familles sont parfois obligées de se contenter d'aliments relativement faciles à cuire. (...) elles se soucient peu, ou ignorent tout, de la manière dont ces changements affectent la qualité d'ensemble de leur

\footnotetext{
${ }^{5}(\ldots)$ in programmes about the prevention of diseases such as malaria and tuberculosis, appropriate local languages would be chosen to reach the targeted communities. (...) They should be recognised for the crucial role they play in the national development at various levels as languages of instruction, communication in agricultural, health and homecraft activities that affect people's lives in rural areas. (Traduction libre, Amélie Baillargeon)

${ }^{6}(\ldots)$ massive contributions could be made to development if local herbalists were equipped with the literacy tools they needed to document and disseminate information on curative practices passed down the generations orally (...). (Traduction libre, Amélie Baillargeon)
} 
alimentation. (p. 59)

Les participants soulignent comme autres conséquences non négligeables de la déforestation, également associables à la surexploitation des terres agricoles, l'érosion des sols et la perte de l'eau, ayant toutes deux une incidence directe sur l'agriculture et la capacité de s'alimenter de manière autonome. Le Green Belt Movement propose comme solutions l'adoption de l'agriculture biologique, la rotation des récoltes, la production alimentaire nationale, le recours aux plantes autochtones et l'incitation à la préservation des savoirs agricoles traditionnels (Maathai, 2001). Enseignées pour la plupart dans le curriculum de l'école bilingue sous forme de savoirs et de savoir-faire locaux, ces solutions peuvent contribuer à outiller les enfants, futurs citoyens qui répondront aux problématiques environnementales. Ariane (22), diplômée de l'école unilingue, acquiesce lorsque nous lui demandons si les anciens de l'école bilingue jouissent d'une meilleure productivité en fournissant cette explication : "Puisque euh... comme eux ils apprennent à faire... à produire les plantes-là... on les montre comment il faut les planter, les arroser pour qu'elles puissent... poussent... pousser bien. ».

\section{ENVIRONNEMENT ET GENRE}

Outre la santé, l'environnement affecte également les femmes dans l'exécution de leurs tâches quotidiennes. En examinant les réponses obtenues des femmes de l'école unilingue ou bilingue aux questions environnementales, nous n'avons pu distinguer de préoccupation environnementale proprement féminine; les hommes semblent en outre aptes à identifier plus de problèmes qu'elles. Néanmoins, les principales préoccupations de celles-ci portent sans conteste sur l'eau et la déforestation.

La collecte d'eau représente la tâche la plus pressante des femmes et souvent, la plus ardue à accomplir compte tenu de divers facteurs, dont la pollution des sources d'eau qu'elles utilisent, le poids de l'eau à transporter et la distance du trajet à parcourir (Roda, 1991). La pression économique pèse aussi lourd sur la collecte de l'eau, notamment pour l'agriculture commerciale, dont les hommes sont les principaux bénéficiaires. En effet, l'intensité de ce type de culture exige une quantité importante d'eau et vide les puits où vont les femmes, les obligeant à la trouver ailleurs, souvent plus loin (Roda, 1991). Confrontées à de telles situations et puisque l'eau est à la base de la plupart de leurs responsabilités familiales, les femmes oublient vite les autres problèmes qui les entourent compte tenu de l'ampleur de ces responsabilités. Pousga (27) est très éloquente à ce sujet: "Nous abandonnons cela en soulevant les seaux (pour aller chercher de l'eau). Tõnd sã n wa z_k so-rãmbã tõnd basda y_nda. ».

Autre facteur désarmant pour les femmes, la déforestation les prive d'une foule de ressources pour s'alimenter, s'abriter et se soigner. Ces ressources, elles les obtenaient autrefois gratuitement; maintenant, elles doivent 
les acheter et perdent ainsi de leur capacité d'achat déjà très faible (Roda, 1991). La cueillette du bois devient d'autant plus délicate puisqu'elle est souvent considérée comme une cause de déforestation (Roda, 1991). En effet, la superficie boisée compte pour $29 \%$ du territoire burkinabè et se situe principalement au sud du pays, le Burkina Faso étant situé dans une zone sahélienne en proie à la désertification (PNUD, 2007, p. 305). Conséquemment, les femmes sont contraintes à piller leur propre environnement pour assurer leur survie quotidienne (Roda, 1991).

Ces constatations sont alarmantes puisque les femmes, les mères de surcroit, sont au cœur d'aspects fondamentaux du développement de l'être humain, notamment la santé, l'éducation et l'alimentation. L'environnement leur sert de principale source pour répondre aux besoins alimentaires de leur famille. indéniable :

Le rôle des femmes relativement à la gestion de l'environnement est

Dans les pays en développement, la relation qu'entretiennent de nombreuses femmes avec l'environnement est vitale dans leur quotidien, par exemple, pour subvenir à leurs besoins en eau, en combustible, en aliments et autres. Ces femmes portent non seulement le poids de la dégradation environnementale, mais jouent également un rôle crucial dans la gestion environnementale. ${ }^{7}$ (Roda, 1991, p. 7)

À force de consacrer tant de temps à la survie et au bien-être de leur famille, les femmes cumulent les connaissances transmises des générations précédentes et acquises au fil de l'exécution des tâches. Ces connaissances, elles les lègueront en héritage social à leurs propres filles. Pour l'eau notamment, elles savent où trouver les sources d'eau locales fiables et de qualité, en plus d'avoir la responsabilité de la collecte de l'eau et du contrôle de son utilisation (Roda, 1991). En tant qu'utilisatrice principale des ressources naturelles et canal de choix pour le transfert de connaissances à la jeunesse, la femme se trouve dans une position privilégiée. En effet, grâce à son expérience et ses aptitudes reconnues officieusement ainsi qu'à son habituelle disponibilité à s'engager dans les actions de développement local, elle a le potentiel de jouer un rôle prépondérant dans la saine gestion de l'environnement et la mise en place de pratiques environnementales durables.

La dimension des femmes est incluse dans tous les programmes de mise en œuvre des conventions sur l'environnement ratifiées par le Burkina Faso depuis le début des années 1990. Chouaïbou Mfenjou (2002) éclaire le discours politique à cet égard en écrivant :

\footnotetext{
${ }^{7}$ In the developing countries, many women's relationship with the environment is vital to their daily lives, for example, in the provision of water, fuel, food and other basic needs. These women not only bear the brunt of environmental degradation, but also play a crucial part in environmental management. (Traduction libre, Amélie Baillargeon)
} 
Les gouvernements auront beau signer toutes les conventions environnementales, rien ne changera en Afrique si les paysans, les femmes, et surtout les jeunes, n'ont d'autres possibilités que d'exploiter la richesse naturelle pour survivre. (p. 35)

Ainsi, bien que le pays des hommes intègres ait apposé sa signature au bas de la totalité des conventions en matière d'environnement, le sort des femmes ne s'améliorera pas tant qu'aucune mesure concrète ne sera prise pour les outiller. Toutes ces connaissances et ces conventions doivent se lier aux besoins économiques locaux, sinon l'atteinte des objectifs de développement humain durable est vouée à l'échec. En outre, Maathai (2001) dit que, tant que les femmes n'auront pas le droit de contrôler leur propre cycle de reproduction, la pression démographique sur l'environnement, ses ressources et la gestion qui en est faite par les familles qui doivent avant tout nourrir les nombreuses bouches qui les composent ne cessera pas :

- DT (cochercheuse) : Mais pour exemple si votre mari veut avoir plus d'enfants que vous, quel serait votre avis.

$L a$ wênd wa yâmb Sàda sâ $n$ dat $n$ paam kamb wùsg $n$ yààg yâmb sên datâ, yâmb tagsg na n yàà wân wâna?

- Yamba (22) : Nous allons suivre le vœu de l'homme.... Parce que nous sommes sa propriété.

Tônd nan tûu raô̂ raab wè. ... Bala yê n so-d la.

Les femmes doivent pouvoir s'exprimer sur les questions d'ordre environnemental auprès des décideurs. Tout organe décisionnel a avantage à tirer parti des connaissances et des aptitudes des femmes, car cette propriété intellectuelle (Rose, 2005) constitue une richesse dont un pays dispose pour améliorer le sort de sa population et favoriser le développement durable à l'échelle locale.

\section{ENVIRONNEMENT ET POLITIQUE}

Omniprésente au Burkina Faso, la structure hiérarchique prédétermine le rôle que chacun doit jouer en fonction de son statut dans la société. Comme l'explique Yves (29), les chefs, le maire et les enseignants ont pour responsabilité de régler les problèmes environnementaux du village et de l'école.

Même si nous voyons la vérité (solution) souvent nous ne pouvons pas sauter pour parler; il faut que nous aussi on se taise pour regarder. $\quad B a$ tônd sâ $n$ ne sidâ wakat ninga tônd ka tôe $n$ yik $n$ long bâmba $n$ na $n$ gom ye; rênna tônd me sind $n$ getê. 
Gardiens de l'environnement, les chefs traditionnels décident des solutions à prendre relativement aux problèmes de leur collectivité. Les chefs et les dirigeants du pays ont beaucoup de pouvoir quant à la gestion des ressources locales. Il n'y a donc rien d'étonnant au fait que six participants disent ne pouvoir rien faire pour participer à la recherche de solutions environnementales. Cette structure hiérarchique traditionnelle a donc une incidence sur la déresponsabilisation environnementale des villageois. Comme l'explique Amélie (23), depuis la mort du chef, les villageois n'ont plus accès à la télévision, car l'électricité est coûteuse et principalement contrôlée par le chef. Les classes sociales déterminent l'accès aux ressources naturelles telles que la terre, l'eau et l'électricité. Au Burkina Faso, le taux d'électrification est de $7 \%$, pourcentage qui indique que, sur un total de 14 millions, 12,4 millions de citoyens n'ont pas accès à l'électricité (PNUD, 2007).

Outre l'accès à l'électricité, l'accès à la terre représente une autre ressource interdépendante à la sphère politique. Seely \& Zeildler (2002) indiquent clairement dans leur article que la distribution et l'occupation des terres reflètent les situations politique, économique et sociale du passé et du présent. Dans le contexte africain, la terre est tantôt sacrée, communautaire et inaliénable, tantôt espace de liberté soumis aux conditions de consommation des membres de la société (Chouaïbou Mfenjou, 2002). Les droits sur le sol se définissent en fonction de l'appartenance à un groupe social et d'un droit traditionnel qui stipule que la terre appartient au premier occupant. Il n'est pas étonnant alors que le problème de répartition des terres ne soit soulevé que par trois hommes et qu'aucune femme n'en fasse mention, car la terre constitue un pouvoir qui revient traditionnellement aux hommes.

Maathai (2001) ajoute qu'en Afrique, la dégradation de l'environnement, la gestion des ressources nationales et le recul de la biodiversité sont principalement liés à la mauvaise gouvernance et la corruption. Cette auteure réitère que : « Nombre d'entre eux (politiciens et décideurs), hélas, n'évoquent la question de la défense de l'environnement qu'en paroles, car ils sont souvent, directement ou indirectement, impliqués dans le pillage des ressources.» (Maathai, 2001, p. 109). Yves (29) adopte une attitude de résignation car, selon lui, peu importe l'effort mis dans une activité, le résultat est tributaire des relations hiérarchiques en place dans le village et commande les comportements à adopter ou à ne pas adopter :

Moi qui suis assis comme cela je ne sais pas ce que je peux faire pour résoudre ce problème. Les maîtres (enseignants) sont là-bas; (...) Sinon je suis petit ici et je vois des problèmes qu'on doit arranger, mais il y a des gens qui sont devant (...), même si nous voyons la vérité souvent nous ne pouvons pas sauter pour parler; il faut que nous aussi on se taise pour regarder. Mam sên zî wotoone wâ yê, mam ka mi mam sên na $n$ maane, tà yell kâng tôog $n$ maneg ye. Karensaam- 
dâmbâ bee beene; (...) « sinon » mam yaa bilf $n$ be ka $n$ ne yèl wùsg sên sègd $n$ manege, la neb $n$ be ka $n$ ya tônd kêem dâmba $n$ le ya tônd saam dâmba (...) ba tônd sâ $n$ ne sidâ wakat ninga tônd ka tôe $n$ yik $n$ long bâmba $n$ na $n$ gom ye; rênna tônd me sînd $n$ getê.

Comme Yves (29), douze autres participants attribuent tout le pouvoir décisionnel aux dirigeants. Selon eux, comme le pouvoir décisionnel revient à cette autorité, ils n'ont ni à participer à la recherche de solutions ni à leur demander d'assumer leurs responsabilités. Ils optent pour l'inaction, car ils se considèrent dépourvus de moyens.

En contrepartie, plusieurs participants à l'étude ne sont pas découragés par la complexité des enjeux. Malgré des contextes politiques local et national défavorables aux initiatives environnementales citoyennes, deux participants de l'école bilingue et un participant de l'école unilingue soulignent l'importance des initiatives locales pour contrer l'inaction de leurs dirigeants. Parmi celles proposées par les participants, on retrouve les associations villageoises. Richard (22) et deux participants de l'école bilingue considèrent qu'en se regroupant en association, les villageois ont plus de chance de trouver des solutions aux problèmes qui minent la vie au village ou encore l'aide pour les régler :

Sinon, nous-mêmes, on peut réunir faire une association (...).

On peut chercher quelque chose qui peut nous aider. Et puis, au moins essayer faire quelque chose contacter des gens qui peuvent nous aider.

Fanon rappelle l'importance de se regrouper pour inventer et discuter ensemble des solutions envisageables pour le milieu : "Les masses doivent pouvoir se réunir, discuter, proposer, recevoir des instructions. Les citoyens doivent avoir la possibilité de parler, de s'exprimer, d'inventer. » (Fanon, 1961, p. 185).

Les langues locales constituent l'outil idéal pour faciliter la participation du plus grand nombre à la recherche de solutions. L'introduction des langues et des activités de production permet l'échange intergénérationnel au sujet des solutions potentielles aux problèmes environnementaux propres à leur collectivité (Jenkins \& Jenkins, 2005). Seules les langues africaines sont en mesure de nommer avec précision la faune et la flore endémiques, les méthodes d'agriculture et les changements propres à l'environnement local (Cajete, 1999). Skutnabb-Kangas (2001) ajoute que :

Les systèmes d'éducation sont les facteurs directs les plus importants de l'assassinat d'une langue. (...) Le droit de l'homme le plus essentiel en matière de langue pour maintenir une diversité linguistique est spécifiquement le droit à un 
enseignement dans sa langue maternelle. ${ }^{8}$ (Skutnabb-Kangas, 2001, p. 201)

Cette auteure est claire à l'effet que la diversité linguistique contribue à la protection de l'écosystème et à la préservation de la biodiversité mondiale. Par l'utilisation des langues autochtones et des savoirs locaux, de même que par la participation des parents d'élèves, l'éducation bilingue est porteuse d'espoir à l'égard de la participation citoyenne relativement aux problématiques environnementales.

\section{ENVIRONNEMENT ET ÉDUCATION}

En plus de l'interdépendance entre environnement et politique, Yoshizumi \& Takaaki (2005), Hanley (2005) et O’Brien (1997b) voient un lien entre le niveau d'instruction et l'importance accordée à la protection de l'environnement. Ils affirment que l'éducation permet la modélisation de comportements respectueux de l'environnement et les discussions sur les problèmes environnementaux. L'école éveille la conscience des gens et leur donne plus d'idées pour résoudre les problèmes :

(...) certains n'ont pas fréquenté (l'école), (...) ils sont dans les ténèbres. (...) si tu es seul avec dix idées, il est bon que tu donnes à d'autres pour que si la personne avait deux idées qu'elles deviennent trois maintenant. (...) La kêer me ka rat tà ba ne tônd sên mi wâ bà tônd zâms bâmb ye. (...)la bâmb sên ket be lik wâ. (...) b yeelame tà fo sâ $n$ tare, $n$ yaa $f$ yembr $n$ tar yam piiga, fo sômb $n$ yâkame $n$ kô nebâ, tà yême paase, sâ $n$ da yaa a yiib bà yême paas tà lebg a tâabo. Roukia (25)

Pratiquement la totalité des hommes ainsi que la grande majorité des femmes voient également dans l'éducation une solution pour l'environnement. Yves (29) considère notamment que : «c'est l'école de nos enfants qui peut résoudre nos problèmes de demain. $\quad b$ modg $n$ ges kamba kareng yelle, tà kamba karengâ $n$ tôe $n$ wa manes tônd yèlâ beoog daare, tà kambâ karengâ yê. ».

Selon l'avis des participants, c'est le curriculum bilingue qui l'emporte sur celui de l'école unilingue. Douze interviewés sur vingt sont unanimes quant aux bienfaits offerts par les enseignements dispensés dans les établissements bilingues. Richard (22) voit dans les techniques enseignées dans le curriculum scolaire de l'école bilingue un avenir assuré pour les diplômés. Mathieu (38) approfondit cette pensée en indiquant que les élèves diplômés de la première cohorte bilingue ont tiré pleinement parti des formations techniques suivies au

\footnotetext{
${ }^{8}$ The educational systems are the most important direct agents of language murder. (...) The most basic linguistic human rights for maintenance of linguistic diversity is specifically the right to mother tongue medium education. (Traduction libre, Amélie Baillargeon)
} 
cours de leur éducation. En effet, celles-ci ont été dispensées dans leur langue et par des personnes-ressources en mesure de leur fournir l'appui requis. En outre, il rajoute que les élèves de l'école bilingue peuvent exécuter leurs tâches quotidiennes à la maison sans avoir à se les faire expliquer, puisque l'apprentissage de ces savoirs et de ces savoir-faire culturels et techniques locaux font partie de leur instruction. Du côté des participantes diplômées de l'école unilingue, Ariane (20) et Julie (22) soulignent que les élèves de l'école bilingue sont mieux outillés à la fin de leurs études pour entreprendre des activités et trouver des solutions.

Parmi les diplômés de l'école bilingue, Rabi (24), Wendyam (20), Zouli (25), Noaga (24) et Pousga (27) évoquent tous le souvenir des animaux offerts par l'école pour soutenir les familles du village. Ces dernières les élèvent et peuvent ainsi profiter des ressources qu'ils procurent. Ouango (23) et Zouli (25) soulignent l'apport indéniable de savoirs et de savoir-faire acquis à l'école bilingue sur les activités agricoles.

Tous les avantages découlant de l'apprentissage des savoirs et des savoir-faire locaux sont au bénéfice des parents qui envoient leurs enfants à l'école bilingue. En effet, en plus d'apprendre dans leur langue maternelle et d'acquérir ultérieurement une autre langue, les enfants baignent dans un univers familier qui naît de leur quotidien, de leur culture et de leurs habitudes de vie. En plus de constituer un investissement à long terme pour les parents, l'éducation prodiguée par l'école bilingue rend les enfants davantage confiants en leurs capacités et plus rapidement autonomes. Yves (29) exprime clairement l'espoir fondé dans les enfants à l'égard des problèmes liés à l'environnement :

(...) fassent tout pour s'occuper de l'école des enfants parce que c'est l'école des enfants qui peut résoudre nos problèmes demain. $B$ modg $n$ ges kamba kareng yelle, tà kamba karengâ $n$ tôe $n$ wa manes tônd yèlâ beoog daare, tà kambâ karengâ yê. ».

Douze des vingt participants, dont sept anciens de l'école bilingue et cinq de l'école unilingue, précisent que l'éducation bilingue est la solution pour résoudre ou, du moins, prévenir plusieurs de leurs problèmes environnementaux. Ils expliquent que, lors des activités de production offertes à l'école bilingue, l'élève apprend grâce aux parents, aux experts locaux et aux enseignants à chercher des solutions aux problématiques locales. L'étude d'Ochala (1991) au Kenya et celle d'Ansell (1999) en Afrique du Sud montrent que les étudiants qui ont reçu une formation technique à l'école sont plus aptes à résoudre des problèmes locaux avec les ressources disponibles dans leur milieu que ceux qui n'en ont pas reçu.

Échanger connaissances, compétences, expériences et idées permet d'accroître la capacité et la confiance d'un peuple à résoudre non seulement des problèmes d'ordre nutritionnel, 
mais aussi d'autres natures liées à la santé et au bien-être. (...) à long terme, les facilitateurs doivent être formés pour analyser les problèmes dans leur contexte local et élargi d'une telle manière qu'ils pourront aider les groupes locaux à élaborer leurs propres façons d'analyser leurs problèmes pour mieux s'attaquer aux sources. ${ }^{9}$ (Ochala, 1991, p. 44)

En plus de l'apprentissage des savoir-faire pratiques, le curriculum de l'école bilingue intègre des connaissances sur les semences, la culture des champs et le reboisement (OSEO, 2003). Bien que ces savoirs ne soient pas systématiquement enseignés à l'école unilingue, certains enseignants, comme celui d'Yves (29), prennent l'initiative d'intégrer l'agriculture ou le reboisement à leurs activités d'apprentissage :

(...) quand nous étions là-bas, nous faisions du jardinage à l'école, des choux, de la salade, un jardin quoi, que nous arrosions et faisions d'autres travaux comme la plantation des arbres dont j'ai parlé. Aujourd'hui, je trouve que la plantation des arbres, c'est quelque chose profite beaucoup; (...) Parce que si on regarde les arbres que nous avons plantés tout de suite pendant notre école, quand il y a une réunion à l'école aujourd'hui, quand les gens arrivent c'est là-bas qu'ils font les réunions. Donc dans ma vie aujourd'hui je sais qu'il profite.

Bon, yaa tà tônd sên dag $n$ be beene wân tônd dag $n$ maana zêed ekollê wâ fâa, choux, salaade, "jardin quoi » $n$ da zâgsdê la d maand tùùm a taab wa mam sên gomd tààsâ selbâ, donc yaa rê la rûnda rûnda mam zî $n$ mi $n$ getê tà tààsâ selbâ, a yaa bûmb sên nafde, sôama ; (...) la ekollê wâ la mam zînd $n$ bâng tùùm bâmb fâa- tà $d$ sâ $n$ na $n$ ges tônd sên sel tààs ninsb meng ne b na ka zâr tônd ekollâ sasa wâ yaa, "reuniuon" sâ n be ekollê wâ rûnda, nebâ sâ n ya yaa tà-bâmb maasmê wâ la b zîn maand $b$ « reunion » râmbâ.

Son propos illustre les bienfaits d'un environnement vert sur la qualité de vie du village et montre que l'école unilingue peut également être porteuse des mêmes bienfaits si les enseignants s'y engagent.

Omolewa (2007) explique que l'hybridité des systèmes éducatifs, en termes d'intégration des savoirs autochtones et «modernes», joue un rôle

\footnotetext{
${ }^{9}$ To share knowledge, skills, experiences and ideas, this will increase people's ability and confidence to solve not only nutritional problems but other problems related to their health and wellbeing. (...) on a long-term basis, facilitators need to be trained to analyse issues in their local and wider context in such a way that they may help grass-root groups develop their own ways of analyzing their problems to be better able to tackle the root causes of problems. (Traduction libre, Amélie Baillargeon)
} 
primordial dans la préservation de la biodiversité. Cajete (1994) rappelle que le cycle d'apprentissage observation-pratique-réflexion respecte les principes de l'éducation autochtone. Dans les principes de l'éducation précoloniale, la nature constitue une ressource pour l'enseignement et le processus d'apprentissage. Ainsi, l'éducation bilingue renoue avec ces principes autochtones, desquels l'enfant apprend de ses parents qui participent aux activités de l'école, de ses enseignants ainsi que des plantes et des animaux qui l'entourent (Omolewa, 2007).

Le curriculum de l'école bilingue permet aux populations locales d'adapter les enseignements aux problématiques locales. Selon Rose (2005), les renseignements que tire l'être humain de son environnement, situé dans un cadre spatio-temporel donné, constituent son bagage intellectuel et par extension, celui de son peuple. Il crée donc un cycle vertueux d'échanges entre les jeunes et les aînés ainsi qu'entre l'école et son milieu.

\section{CONCLUSION}

La majorité des participants considèrent que l'enfant diplômé de l'école bilingue deviendrait un citoyen doté d'une conscience verte plutôt qu'un observateur identifiant les problèmes sans pouvoir les résoudre. Il est vrai que l'instruction en général comporte des bienfaits pour l'environnement, mais l'éducation bilingue ajoute cette prise en charge des ressources locales grâce à la participation des parents aux activités de production. Bien que l'éducation bilingue ait mise en branle le processus de formation de la conscience verte, beaucoup de choses restent à faire pour que celle-ci se traduise dans le mode de vie des diplômés une fois rendus à l'âge adulte.

En conclusion, les problèmes environnementaux identifiés par les participants sont, en ordre d'importance : l'accès à l'eau, la déforestation, la répartition des terres, le manque d'infrastructures sanitaires et routières et la gestion des déchets. Parmi les participants qui identifient des problèmes, nous ne remarquons pas de différence entre les préoccupations environnementales relevées par les anciens des écoles bilingue et unilingue.

Du côté des solutions proposées, l'inaction causée par le statut hiérarchique des rôles dans le village, le regroupement associatif, la demande d'aide, l'éducation en général et l'éducation bilingue constituent les principales catégories de réponses données par les participants.

Environnement, santé et femmes sont trois éléments intrinsèquement liés au développement durable humain. Le premier constitue le berceau nourricier et protecteur de l'être humain; le deuxième, son moteur pour effectuer ses activités quotidiennes; et le troisième, les gardiennes du bien-être et de l'éducation de l'être humain ainsi que les protectrices de l'environnement, parce que principales utilisatrices à des fins familiales. Ainsi, pour qu'il y ait développement durable, ces trois éléments doivent être bien équilibrés pour 
éviter d'induire un comportement de survie chez l'être humain, car c'est ce type de comportement qui engendre une vision à court terme et fait oublier les besoins des générations futures puisque ceux du lendemain ne sont pas assurés.

En effet, les questions d'ordre environnemental sont multidisciplinaires et étroitement liées aux questions de genre, de santé, de politique et d'éducation. L'éducation environnementale n'est pas qu'une affaire scolaire, elle doit s'étendre au-delà des murs des établissements d'enseignement pour créer non plus une conscience verte individuelle, mais plutôt une conscience verte collective. L'éducation environnementale ainsi que les politiques environnementales doivent être analysées dans leur ensemble; autrement, elles perdent tout leur sens. Sans l'adoption d'une approche holistique durable, les citoyens seront toujours contraints à combler leurs besoins vitaux quotidiens sans préserver, voire développer, leur environnement : « Nous abandonnons cela en soulevant les seaux. Tõnd sã $n$ wa zẽk so-rãmbã tõnd basda yẽnda.» (Pousga (27)). Maathai (2001) recommande que les curricula scolaires d'éducation civique soient enrichis d'un volet spirituel en lien avec l'environnement :

La réhabilitation de valeurs spirituelles et culturelles positives est chose importante, car elles contribuent à la restauration de la confiance en soi et de l'identité personnelle. Cela permet également de protéger la biodiversité, les connaissances, les pratiques et la sagesse traditionnelle. (p. 72)

Cette même auteure recommande également que la conscience verte fasse partie $\mathrm{du}$ programme de formation des enseignants. Nous croyons que les recommandations de Maathai (2001) seraient bénéfiques pour toutes les écoles burkinabè. Lorsqu'on regarde ce qui existe déjà dans les écoles bilingues en termes d'éducation écologique, il serait souhaitable que les activités de production, qui consistent principalement à exploiter les ressources premières, soient jumelées systématiquement à des activités de préservation de l'environnement. 


\section{Références}

Agyeman, J. (2005). Sustainable communities and the challenge of environmental justice. New York: New York University Press.

Ansell, N. (1999). Life chances and education in Southern Africa. In M. Redclift (Ed.), Sustainability: Life chances and livelihoods (pp. 144-157). London: Routledge.

Babin, R., \& Vaillancourt, J.-G.

Benneh, G., Morgan, W. B., \& Uitto, J. I. (1996). Sustaining The Future : Economic, Social, and Environmental Change in Sub-Saharan Africa. New York: United Nations University Press.

Cajete, G. (1994). Look to the mountain: An ecology of indigenous education. Skyland, NC: Kivaki press.

Cajete, G. (1999). A people's ecology: Explorations in sustainable living. Santa Fe, NM: Clear Light.

Chouaïbou Mfenjou, M. (2002). L’Afrique à l'épreuve du développement durable. Paris: L'Harmatan.

Dei, G. J. S. (2006). Mapping the terrain: Towards a new politics of resistance. In G. J. S. Dei \& A. Kempf (Eds.), Anti-colonialism and education: The politics of resistance (pp. 1-24). Rotterdam, Netherlands: Sense.

Fanon, F. (1961). Les damnés de la terre. Paris: La Découverte.

Hanley, P. (2005). Holistic yet tangible: Embracing the challenge of complexity for education for sustainable Development. Current Issues in Comparative Education, 7(2), 85-93.

Jenkins, K. A., \& Jenkins, B. A. (2005). Education for Sustainable Development and the Question of Balance: Lessons from the Pacific. Current Issues in Comparative Education, 7(2), 114129.

Kibora, L., Napon, A., Batiana, A., \& Belemgoabga, P. (1999). Étude de cas de cinq types d'écoles communautaires au Burkina Faso. Ouagadougou, Burkina Faso: UNESCOOuagadougou.

Maathai, W. (2001). Pour l'amour des arbres. Paris: L'Archipel.

Moyo, T. (2003). The democratisation of indigenous languages: The case of Malawi. AILA Review, 16, 26-37.

O’Brien, C. (1997a). Education for Sustainable Community Development. Barefoot College, Tilonia, India.

O’Brien, C. (1997b). Education for sustainable community development., Barefoot College, Tilonia, India.

Ochala, P. A. (1991). Nutrition education. In S. Van der Vyncht \& M. Sachs-Israël (Eds.), Curriculum reorientation in rural development implications for home economics (pp. 4150). Nairobi, Kenya/Paris: Kenyatta University/UNESCO.

Omolewa, M. (2007). Traditional African modes of education: Their relevance in the modern world. International Review of Education, 53(5-6), 593-612.

Omoniyi, T. (2003). Local policies and global forces: Multiliteracy and Africa's indigenous languages. Language policy, 2, 133-152.

OMS (2006). Core health indicators in Burkina Faso Retrieved 2 juin, 2008, from http://www.who.int/whosis/database/

Orr, D. (1992). Ecological literacy: Education and the transition to a postmodern world. New York: State University of New York Press.

OSEO (2003). Programme d'éducation bilingue au Koulpelogo : rapport d'activités pour la période du ler janvier au 31 décembre 2002. Ouagadougou, Burkina Faso: OSEO.

PNUD (2007). Human development report. New York: Oxford University Press. 
Roda, A. (1991). Women and the Environment. London: Zed Books.

Rose, D. (2005). An indigenous philosophical ecology : Situating the human. The Australian Journal of Anthropology, 16(3), 294-305.

Seely, M., \& Zeidler, J. (2002). Land distribution and sustainable development. In V. Winterfeldt, T. Fox \& P. Mufune (Eds.), Namibia Society Sociology. Windhoek, Namibia: Reader in Namibian Sociology.

Shiva, V. (1989). Forestry crisis and forestry myths : A critical review of tropical forests : A call for action. Penang, Malaisie: World Rainforest Movement.

Skutnabb-Kangas, T. (2001). The globalisation of (educational) language rights. International Review of Education, 47(3-4), 201-219.

Yoshizumi, M., \& Takaaki, M. (2005). Realizing education for sustainable development in Japan: The case of Nishinomiya City. Current Issues in Comparative Education, 7(2), 104-113.

Constance Lavoie est professeure associée au Département des sciences de l'éducation à l'Université du Québec à Chicoutimi. Ses recherces portent sur l'enseignement du français en milieu autochtone. (constance lavoie@uqac.ca)

Amélie Baillargeon est traductrice et étudiante en nutrition à l'Université de Montréal (amelie.baillargeon@umontreal.ca) 